\title{
The Psychological Nursing Interventions Based on Pygmalion Effect Could Alleviate Negative Emotions of Patients with Suspected COVID-19 Patients: a Retrospective Analysis
}

\author{
Sisi Zhang' \\ Yazi Liu ${ }^{2}$ \\ Shuning Song ${ }^{3}$ \\ Shixiong Peng ${ }^{4}$ \\ Mao Xiong ${ }^{5}$ \\ 'Nursing Department, Hunan Provincial \\ People's Hospital/The First Affiliated \\ Hospital of Hunan Normal University, \\ Changsha, Hunan Province, People's \\ Republic of China; ${ }^{2}$ Pediatric Intensive \\ Care Unit, Hunan Provincial People's \\ Hospital/The First Affiliated Hospital of \\ Hunan Normal University, Changsha, \\ Hunan Province, People's Republic of \\ China; ${ }^{3}$ Geriatric Department, Hunan \\ Provincial People's Hospital/The First \\ Affiliated Hospital of Hunan Normal \\ University, Changsha, Hunan Province, \\ People's Republic of China; ${ }^{4}$ The First \\ Affiliated Hospital of Hunan Normal \\ University/Hunan Provincial People's \\ Hospital, Changsha, Hunan Province, \\ People's Republic of China; \\ ${ }^{5}$ Ophthalmology and Stomatology \\ Department, Hunan Provincial People's \\ Hospital/The First Affiliated Hospital of \\ Hunan Normal University, Changsha, \\ Hunan Province, People's Republic of \\ China
}

Correspondence: Shixiong Peng;

Mao Xiong

Tel +86 |3|656|603|:

$+86|9973| 19154$

$\mathrm{Fax}+86073183929235$

+86073183929236

Email1532808066@qq.com;

704305468@qq.com
Purpose: This study aims to explore the psychological status of suspected COVID-19 patients during quarantine and put forward a new yet effective psychological nursing strategy for intervention.

Patients and Methods: We performed a retrospective study with suspected COVID-19 patients who were hospitalized to the two hospitals of Hunan province, China and accepted the intervention of psychological nursing from $01 / 2020$ to $03 / 2020$. The control group received routine psychological nursing care and the observation group received the new psychological nursing intervention according to Pygmalion effect.

Results: A total of 89 objects were included in the analysis. Results of the questionnaire before intervention showed that the majority of isolated suspected COVID-19 patients showed negative emotions, with the incidence of depression $(51.69 \%)$, anxiety $(14.617 \%)$, inverted provocation $(22.47 \%)$, extraverted provocation $(25.84 \%)$. And the extraverted provocation scores of female patients was significantly higher than that of male counterparts $(\mathrm{P}<0.05)$. At discharge, compared with the control group, the scores of depression, anxiety, introversion and extraversion of patients in the observation group were significantly lower after nursing intervention based on Pygmalion effect. The satisfaction rate of psychological care based on Pygmalion effect was $86.66 \%$.

Conclusion: Suspected COVID-19 patients tend to show the symptoms of depression, anxiety and irritation during quarantine. The psychological nursing based on Pygmalion effect is helpful to alleviate their negative emotions.

Keywords: COVID-19, suspected patients, Pygmalion effect, psychological care, depression, anxiety

\section{Introduction}

Severe acute respiratory syndrome coronavirus 2 (sars-cov-2) had rapidly spread all over the world and developed into a pandemic. ${ }^{1}$ The World Health Organization (WHO) announced the new name of the disease: coronavirus disease (COVID-19). ${ }^{2}$ As of April 30, 2020, more than 3 million COVID-19 cases have been confirmed in more than 100 countries and regions. ${ }^{3}$ So far, the rapid spread of sars-cov- 2 has caused considerable harm to public health and economy. ${ }^{4}$

The infectious disease epidemic situations caused by COVID-19 is a sudden public crisis. It not only poses a serious threat to people's physical health, but also 
brings strong psychological impact to the masses, resulting in corresponding psychological and behavioral problems. $^{5-7}$ COVID-19 cases are prone to negative emotions, such as anxiety, depression, fear, irritability, especially during the 14 days of isolation observation. ${ }^{8,9}$ This brings a burden to the society and leads to the decline in their life quality and treatment compliance. Therefore, the need for psychological intervention is urgent. However, there is few researches in this field.

The Pygmalion effect is a psychological effect summarized by American psychologist Rosenthal, who believes that positive suggestions, encouragement, trust and expectation can enhance people's self-worth, obtain positive motivation and strive to reach the expected value. $^{10,11}$ Initially, it was mainly used in education and teaching. In recent years, it has been gradually used in clinical intervention to alleviate patients' negative emotions and improve treatment compliance. ${ }^{12}$ This study developed a new nursing program based on Pygmalion effect and explored its affect on psychological intervention of suspected COVID-19 patients in isolation period. It will offer a new perspective for the psychological nursing of COVID-19 patients in isolation period.

\section{Materials and Methods}

\section{Study Design and Objects}

This study is a multi-center, retrospective study conducted at the Hunan Provincial People's Hospital (the First Affiliated Hospital of Hunan Normal University) and The First Affiliated Hospital of University of South China. And this work was approved by the Ethics Committee of Hunan Provincial People's Hospital/The First Affiliated Hospital of Hunan Normal University (2020 Scientific Research Ethics Review NO: 04), and conducted in accordance with the ethical guidelines of Declaration of Helsinki.

According to the following criteria, two investigators independently collected the data of patients who were suspected COVID-19 during 01/2020-03/2020 from the medical records management system, including age, sex, occupation, educational level, family location, and results of questionnaire survey. Then the data was crosschecked, and if there were any differences, consult a third investigator. ${ }^{13}$ Inclusion criteria: (1) Meet the diagnostic criteria of suspected cases in the "Diagnosis and Treatment Plan for COVID-19" issued; ${ }^{14}$ (2)Education above elementary school, with good communication skills; (3) Informed consent to participation in this study. Exclusion criteria: (1) Confirmed cases of COVID-19; (2)History of mental illness; (3)Cancer and other serious diseases. (The minimum sample size was calculated as 88 , considering the type I error rate $=0.05$ and power $=90 \%$ (the $\mathrm{G}^{*}$ Power tool was used for calculations)). ${ }^{15,16}$

Suspected COVID-19 patients began to receive psychological intervention in 2 individual isolation wards after admission, once a day, 20-30 minutes each time. The control group received regular psychological care, including empathy, comfort, question and answer, and diversion. The observation group was added psychological nursing care based on the Pygmalion effect on the basis of the intervention of control group (detail as follows). Questionnaires were used before and after the intervention.

\section{The Psychological Nursing Interventions Based on Pygmalion Effect Establishment of Nursing Group}

The nursing group was composed of 6 nurses who had been trained in the Pygmalion effect (2h/day, 2 weeks) and had the qualifications of psychological counselors. A deputy director of the Nursing Department, a national second-level psychological counselor, was mainly responsible for guiding plan design and activity coordination in this event; 1 head nurse in the isolation ward and 3 primary nurses are mainly responsible for the implementation of the activity. Taking three shifts a day, 8 hours a shift working mode.

\section{Creating a Good Atmosphere}

The members of the team communicated with the patient to understand the patient's psychological needs, the source of negative emotions, and the specific factors that affected emotions. In the ward, hung up the reminder to wear a mask, 7 correct steps to wash hands, the 48-character code for the new coronavirus, the publicity pictures for the prevention and control of the COVID-19, and encouragement slogans such as the fight against the "epidemic"; played targeted mindfulness decompression audio, and used modern communication methods to timely input the care, comfort, encouragement and other emotional support from relatives to create a positive humanistic environment in the isolation ward.

\section{Delivering Expectations and Internalizing Expectations}

Nursing group members integrated the Pygmalion effect concept into emotional management, focusing on 
strengthening isolated patients' cognition of the COVID-19 in order to change their negative views, and regulate their own emotions. (1)Longing and expecting. Head nurses and primary nurses told patients about the epidemiological characteristics, clinical manifestations, latest epidemic developments, disease prognosis and treatment plans of the COVID-19; and the purpose of isolation; guided patients to protect themselves, such as wearing masks and correcting the way they wash their hands; informed patients of the successful cases of healing to help them build confidence and courage to fight the disease, and enhance the patient's vision and expectations for curing the disease. (2)Behaviours and feelings. The responsible nurse approached the patient, listened carefully to the patient's complaint, created an atmosphere for the patient to vent bad emotions, and encouraged the patient through eyes and body language, such as tapping the patient's shoulders and back, to eliminate the patient's fear and helplessness. At the same time, the patient was guided to carry on meditating and relaxation. First, the patient was asked to close his eyes and feel the inner feelings, and then was guided by the nurse to imagine the scenes of his future life with a relaxed, confident and firm belief, and finally was invited to share their inner emotions. During this process, the patient's feelings and emotional changes were recorded, and they were given affirmation, praise and encouragement during the sharing process. (3)Acceptance and internalization: Under the guidance of the group members, the patients were encouraged to use self-motivation methods such as positive "self-talk", saying to themselves "I did a good job!" to affirm their own efforts and achievements, such as actively cooperating with the treatment, ensuring adequate sleep; enjoying solitude by reading a good book, listening to a piece of beautiful music, watching a good inspirational drama etc. Psychological suggestion: positive "self-talk" as well as other methods to control negative emotions could help patients gradually accept isolation, and establish expectations of overcoming the disease. ${ }^{17}$

\section{Information Feedback}

Patients still need to do home isolation after discharge. The following measures are targeted to evaluate the behavior of discharged patients in a timely and accurate manner for the purpose of deciding whether to maintain or adjust expectations: (1) Strengthen the health guidance of discharged patients: instructing patients to continue selfhealth monitoring after discharge; supervising patients to

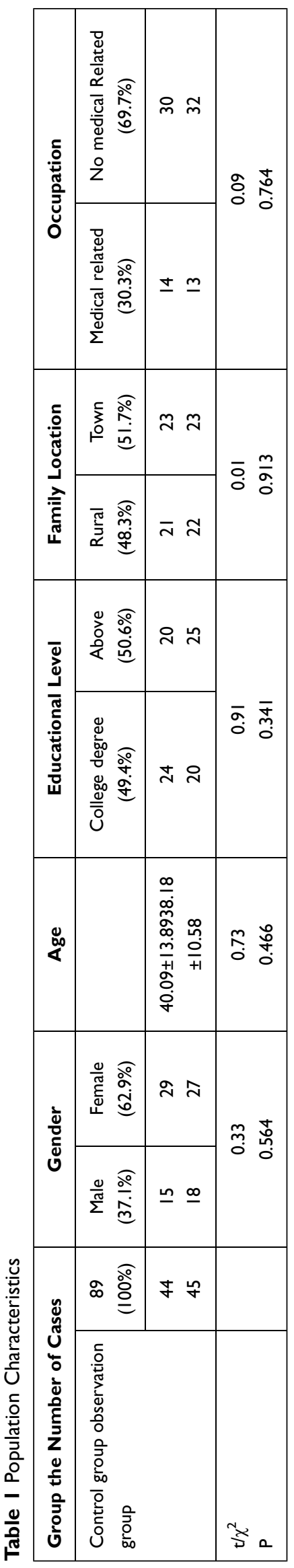


actively and promptly report personal information and health status through various means, and guiding patients to do self-management after discharge, such as wearing masks, doing hand hygiene, and reducing close contact with family members and outings, etc. (2) Make full use of communication technology to do follow-up visits. The nursing group members are able to timely discover and deal with possible physical and psychological problems of patients via WeChat, telephone return visits, family doctors signing App and other means to interact with discharged patients' information. (3) Develop five measures for home isolation, including making a plan, arranging the things to do every day, especially ensuring regular diet and adequate sleep; learning a new skill and enjoying the process; talking to family members and friends through video, phone calls, etc. and seeking professional support through various media when they are not available and self-adjustment is difficult; taking a daily workout, do your favorite indoor sports or use fitness apps to exercise remotely with friends; considering it a specific experience that you obtain valuable life experience from it, and thinking about what kind of life attitude should be possessed to face work, life and study in the future.

\section{Questionnaire Survey} Irritating, Depression and Anxiety Status

The irritation, depression, and anxiety scales (Irritability, Depression and Anxiety Scale, IDA) ${ }^{18}$ were used to evaluate the emotional status of two groups of patients within 12 hours after admission and 12 hours before discharge. IDA included depression, anxiety, introverted agitation, and extroverted agitation, a total of 18 items, with a scale of 0 to 3. The higher the score, the greater the degree of depression, anxiety, and agitation. Depression factor $<4$ is considered normal, 4-6 points are marginal, $>6$ is abnormal; anxiety factor $<6$ is normal, $6-8$ is marginal, $>8$ is abnormal; introverted stimulus factor $<4$ is normal, 4 to 6 points edge, $>6$ points to abnormal; extroversion stimulus factor $<5$ points to normal, 5 to 7 points edge, $>7$ points to abnormal. The scale has good reliability and validity. ${ }^{19}$
A
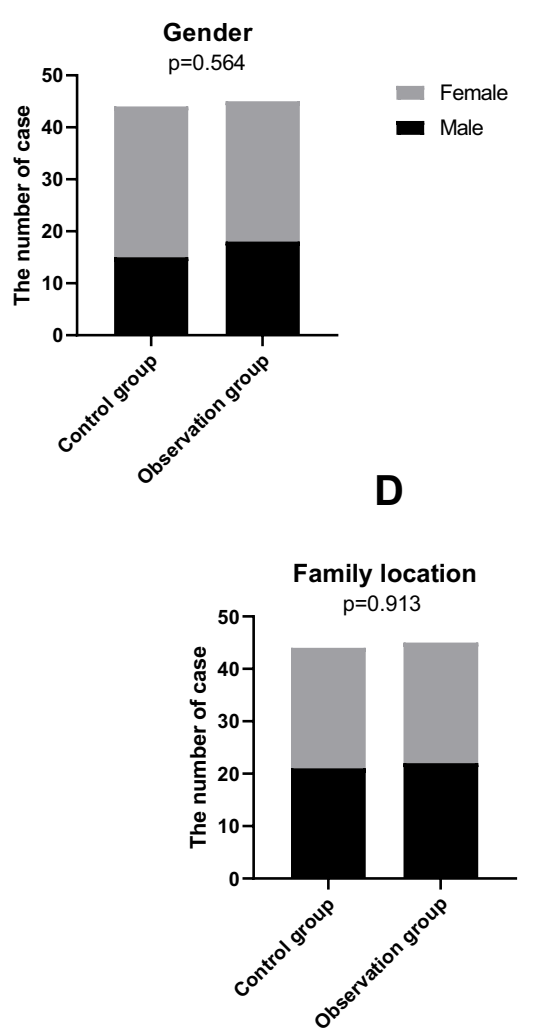

B

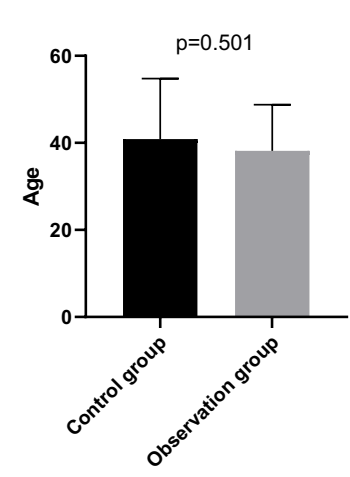

C

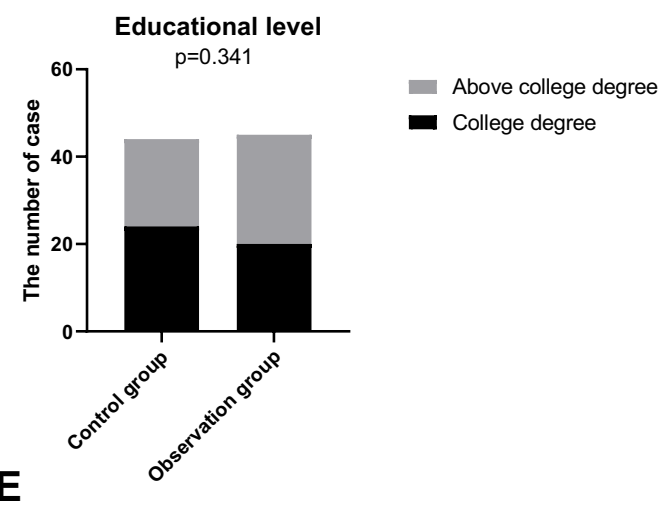

Figure I Population characteristics of control group and the observation group suspected COVID-I9 patients. (A) Gender; (B) age; (C) educational level; (D) family location; (E) occupation.
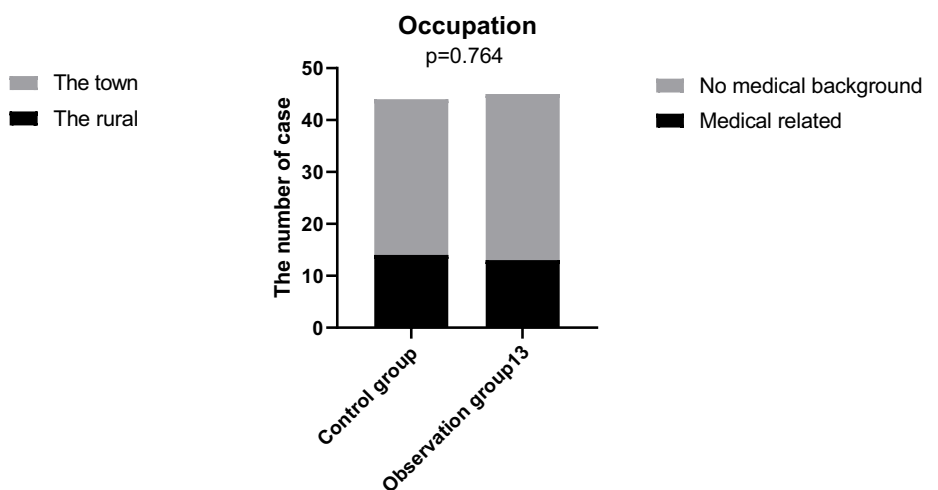
Table 2 The Incidence Rate of Negative Emotions in Suspected COVID-19 Patients During Isolation

\begin{tabular}{|l|c|c|}
\hline Item & The Number of Cases & Rate \\
\hline Totality & 89 & $100 \%$ \\
Depression & 46 & $51.69 \%$ \\
Anxiety & 13 & $14.61 \%$ \\
Inverted provocation & 20 & $22.47 \%$ \\
Extraverted provocation & 23 & $25.84 \%$ \\
\hline
\end{tabular}

\section{Evaluation of Psychological Nursing Activities}

The self-made psychological nursing activity evaluation questionnaire was used to evaluate the psychological nursing activity based on the Pygmalion effect, which was filled out by the observation group at the time of discharge. The questionnaire included 10 items, with $1-10$ points from extremely non-conforming to extremely conforming. The higher the score, the higher the satisfaction, and the 9 points was set as cut-off. ${ }^{20}$

\section{Statistical Analysis}

SPSS19.0 (IBM, Chicago, IL, USA) software ${ }^{21}$ was utilized for statistical description. The $\chi 2$ test was used for comparisons among the categorical variables and independent $t$-test to compare the continuous variables, and the test level is $\alpha=$ $0.05, \mathrm{P}<0.05$ was considered statistically significant.

\section{Results}

\section{Population Characteristics}

A total of 89 objects were included in the analysis (control group 44 and observation group 45). Among of the 89 participants, the majority were women (62.9\%); most were middle-aged; most had a bachelor's degree $(50.6 \%)$; most occupations were not related to medicine (69.7\%) (Table 1). However, there were no significant differences between the observation group and the control group in gender, age, education level, residence and occupation (Figure 1).

\section{Emotions of Suspected COVID-19 Patients Tend to Be Negative and Psychological Nursing Based on Pygmalion Effect Can Effectively Alleviate Them}

A total of 89 valid questionnaires were collected (response rate of $100 \%$ ). Scale test results for all participants indicated that most of the suspected COVID-19 patients in isolation were depression $(51.69 \%)$, anxiety $(14.617 \%)$, inverted provocation $(22.47 \%)$ and extraverted provocation $(25.84 \%)$ (Table 2). And the extraverted provocation score of female patients was significantly higher than that of male counterparts $(\mathrm{P}<0.05)$ (Table 3, Figure 2). This phenomenon suggests that psychological problems are common among suspected COVID-19 patients in isolation. In addition, there were no differences in depression, anxiety, introverted and extraverted irritations between suspected COVID-19 patients in the pre-intervention treatment group and the control group. However, after the psychological nursing based on Pygmalion effect, the psychological status of depression, anxiety, inward and outward irritations of suspected COVID-19 patients in the observation group were significantly relieved compared with the control group (Table 4, Figure 3).

\section{Patients in the Observation Group Were Satisfied with the Psychological Nursing Activities Based on Pygmalion Effect}

Patients in the observation group were given 10 items to evaluate psychological nursing activities based on Rosenthal effect, and the average score of each item was more than 8 points, indicating that psychological nursing activities based on Pygmalion effect had a positive impact on patients. The patient satisfaction score was $9.20 \pm 0.72$, and the satisfaction rate was $91.01 \%$, suggesting that psychological nursing activities based on Rosenthal effect were highly accepted among suspected COVID-19 patients in isolation period (Table 5).

Table 3 The Comparison of the Scores of Negative Emotions Between Female and Male During Isolation

\begin{tabular}{|l|c|c|c|c|}
\hline Gender & Depression & Anxiety & Inverted Provocation & Extraverted Provocation \\
\hline Female & $7.48 \pm 2.77$ & $6.89 \pm 1.87$ & $6.20 \pm 1.51$ & $7.38 \pm 2.65$ \\
Male & $6.94 \pm 2.40$ & $6.55 \pm 1.91$ & $5.67 \pm 1.79$ & $6.21 \pm 1.93$ \\
$t / P$ & $0.94 / 0.351$ & $0.84 / 0.402$ & $1.49 / 0.140$ & $2.20 / 0.030$ \\
\hline
\end{tabular}


A

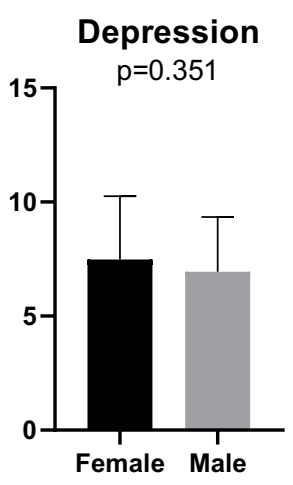

C
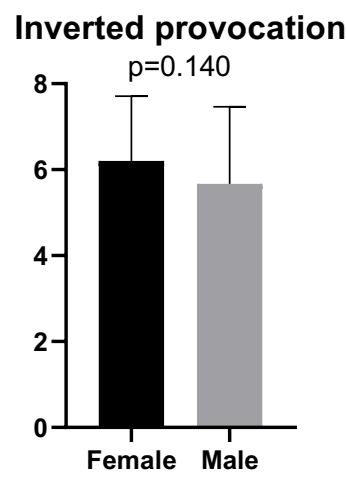

B

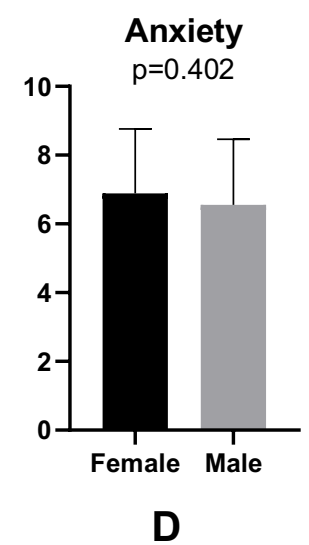

Extraverted provocation

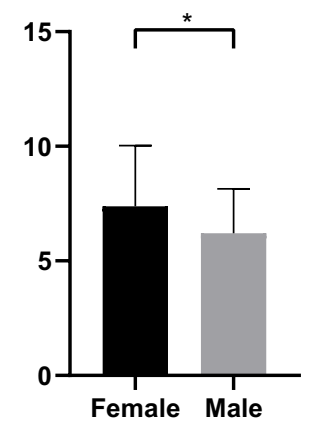

Figure 2 The comparison of the scores of negative emotions between female and male during isolation. (A) Depression; (B) Anxiety; (C) Inverted provocation; (D) Extraverted provocation. $* \mathrm{P}<0.05$.

\section{Discussion}

The public often have different degrees of psychological stress in the face of sudden infectious public health incidents, especially in the high-incidence period of infectious diseases, they are prone to various negative emotions. ${ }^{22,23}$ In the research of D'Emeh W. et al, COVID-19 scale and self-testing anxiety scale were collected from 240 nurses. They found that they had experienced tremendous pressure and anxiety related to work under the background of COVID-19 pandemic. $^{24}$ Sandín B. et al found that 144 adolescents were investigated (aged 12-18, 55 boys $(38.2 \%)$ and 89 girls $(61.8 \%))$, most of whom showed a higher level of anxiety and depression symptoms during the COVID-19 pandemic. $^{25}$ It is even possible to change people's lifestyles under such intense psychological pressure, for example, women are more likely to eat to cope with stress. ${ }^{26,27}$ Therefore, nursing intervention is of great importance to negative emotions caused by COVID-19. Pygmalion effect has been more and more widely used in clinical nursing in recent years. ${ }^{28,29}$ Therefore, this study

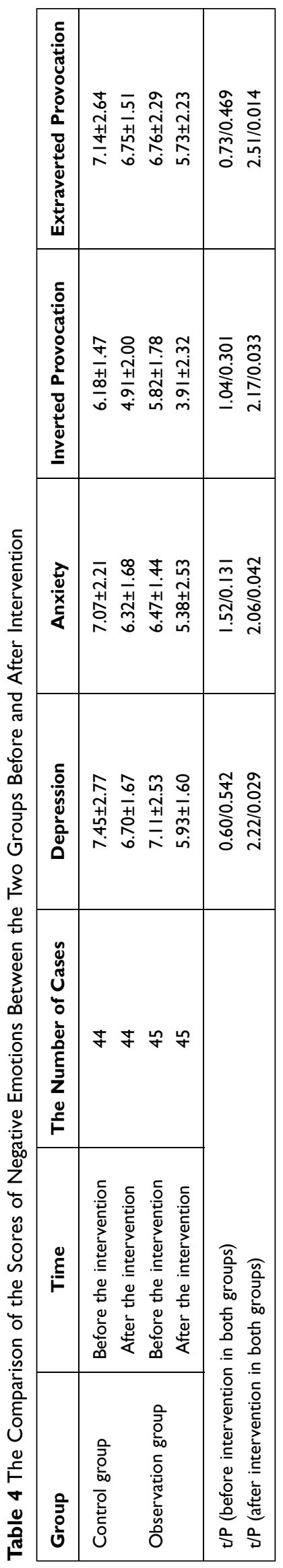




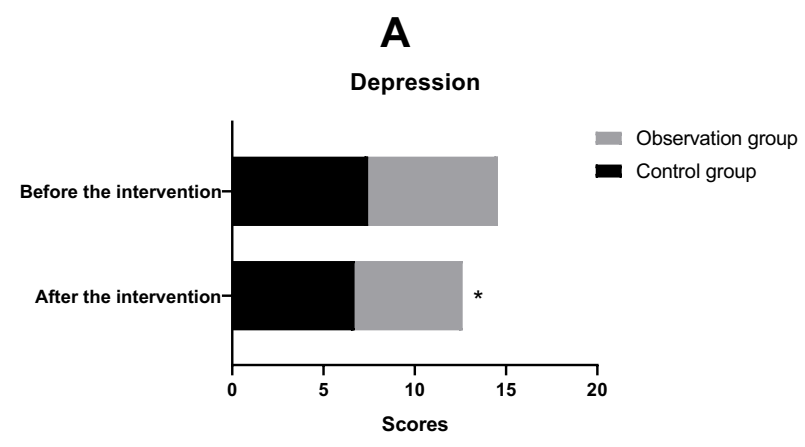

C

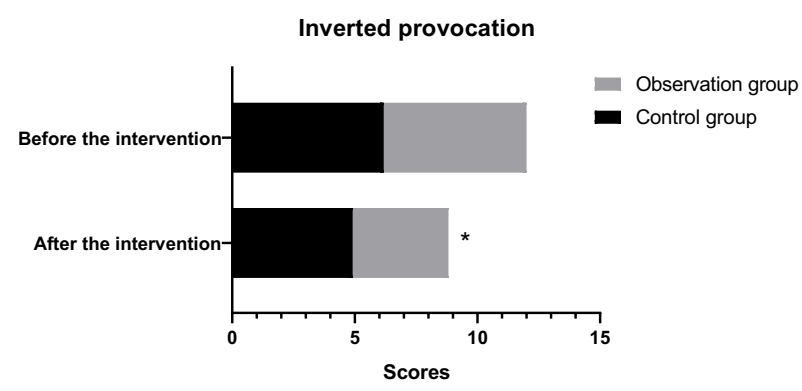

B

Anxiety

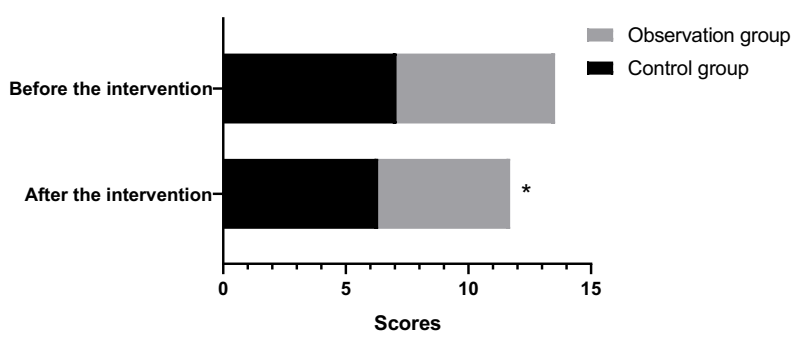

D

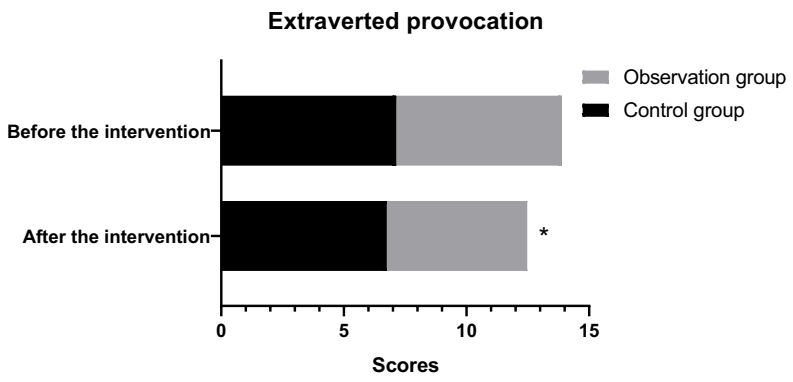

Figure 3 The comparison of the scores of negative emotions between the two groups before and after intervention. (A) Depression; (B) anxiety; (C) inverted provocation; (D) extraverted provocation. *P $<0.05$.

explored the effect of new intervention on patients with new crown pneumonia from the perspective of psychological nursing based on Pygmalion effect.

The results of this study showed that the depression, anxiety, introversion and extroversion stimulus scores of the two groups of suspected infected patients were all in an "abnormal" state when they were admitted to the hospital, which indicate that the COVID-19 patients in isolation period generally have negative emotions. It may be due to: (1) subjective perception. COVID-19 is highly contagious and there is no specific treatment, which has increased people's tension. ${ }^{15}$ Meanwhile, the speed of information dissemination on modern social networks is fast, and many false information can easily cause panic among the people. ${ }^{30}$ In addition, there was the fear from one's heart, the fear of infecting his/her family members and/or afraid of losing his/her job. (2) The objective environment. Being treated in isolation in a single room and in a closed space for a long time, patients are more likely to produce negative emotions due to lack of communication

Table 5 The Evaluation Score of Psychological Nursing Activity Based on Pygmalion Effect

\begin{tabular}{|l|c|}
\hline Entry & Score $^{\#}$ \\
\hline Learned to regulate my bad emotions & $8.02 \pm 1.00$ \\
Learned about COVID-19 & $8.13 \pm 0.93$ \\
Learned how to better deal with illness & $8.20 \pm 0.81$ \\
I have new expectations for my future life & $8.16 \pm 0.84$ \\
Taking part in this activity makes me more and more confident & $8.04 \pm 1.33$ \\
Trust and honesty can be achieved in activities & $8.13 \pm 1.34$ \\
Being willing to share my inner thoughts in activities & $8.04 \pm 1.03$ \\
Be willing to share your opinions with others & $8.02 \pm 0.98$ \\
I like the way of this activity & $9.02 \pm 0.77$ \\
Satisfied with this activity & $9.20 \pm 0.72$ \\
Satisfaction rate & $91.01 \%$ \\
\hline
\end{tabular}

Note: ${ }^{\#}$ The total score is 10. 
and worries about the disease. ${ }^{31}$ Furthermore, by gender grouping, we found that the extraverted provocation score of female patients was significantly higher than that of male ones, suggesting that women might be more prone to negative emotions during the COVID-19 epidemic. This is consistent with previous research, which women were more likely to suffer from psychological stress during and after the recent pandemic. ${ }^{32}$ However, after given Pygmalion effect psychological care based on the above, the observation group had significantly lower scores of depression, anxiety, introversion and extroversion than the control group, indicating that the Pygmalion effect Psychological care can effectively alleviate the negative emotions of suspected COVID-19 patients. Our study brought a new strategy for the psychological treatment of COVID-19 patients in isolation period.

Psychological care of patients needed to promote the patient's understanding of the disease and pay attention to the patient's psychological feelings. ${ }^{33}$ The result of the evaluation of psychological nursing activities showed that the patients could feel the effects of subjective efforts, transform passive behaviors into active behaviors, strengthen self-affirmation, and enhance patients' selfregulation and self-management capabilities by Pygmalion effect psychological nursing. The follow-up visits were equally important in the psychological care when the patients were at post-discharge. ${ }^{34}$ Psychological interventions conducted through modern information methods could continuously track the emotional changes of the patients, help the patients adjust their mental health in time, and play a dynamic and continuous role in alleviating negative emotions. In addition, the evaluation of observation group's psychological care activities based on the Pygmalion effect was above 8 points, and the overall satisfaction with the activities was 9.17 points, indicating that the patients recognized and were satisfied with the psychological care activities. Psychological care based on the Pygmalion effect allows patients to realize that hard work is rewarding, which constantly motivates patients to actively share and regulate emotions. Alleviated negative emotions through continuous intervention contributes to patients' gradual acceptance of the isolation, hence their feeling full of hope towards the future.

\section{Implications}

In the context of the COVID-19 pandemic, there are suspected COVID-19 patients in isolation every day. ${ }^{35}$ During the 14-day isolation period, they experienced a high level of stress due to uncertainty about the nucleic acid results. ${ }^{36}$ Given this situation, we aim to provide evidence of the psychological status of suspected COVID-19 patients in isolation. It calls for improvement not only in the physical health but also mental health of patients. Moreover, our study provides an intervention strategy for negative emotions in patients with suspected COVID-19 in isolation.

\section{Limitations}

This study still has some limitations. This study is not a prospective research. The number of cases in this study is small, and there may be some bias. In the later stage, a larger sample and long-term study is needed and we will further explore the effect of nursing intervention based on Pygmalion effect on the prognosis of COVID-19.

\section{Conclusion}

Suspected COVID-19 patients tend to suffer from depression, anxiety and irritating during quarantine. The psychological nursing based on Pygmalion effect is helpful to alleviate the negative emotions of patients with suspected COVID-19 and promote patients' confidence and courage to face diseases.

\section{Data Sharing Statement}

The authors of included studies should be contacted individually regarding requests to share individual patient data.

\section{Acknowledgments}

The authors acknowledge the contributions of Professor Huiqing Wang the First Affiliated Hospital of University of South China and Professor Hao Feng Hunan Provincial People's Hospital/The First Affiliated Hospital of Hunan Normal University for their helpful suggestions on this work.

\section{Funding}

This study was supported by the Hunan Provincial Health Commission directs scientific research projects (202114051780).

\section{Disclosure}

The authors have disclosed no potential conflicts of interest, financial or otherwise. 


\section{References}

1. Karim SSA, Karim QA. Omicron SARS-CoV-2 variant: a new chapter in the COVID-19 pandemic. Lancet. 2021;398(10317):2126-2128. doi:10.1016/S0140-6736(21)02758-6

2. Zhou F, Yu T, Du R, et al. Clinical course and risk factors for mortality of adult inpatients with COVID-19 in Wuhan, China: a retrospective cohort study. Lancet. 2020;395(10229):1054-1062. doi:10.1016/S0140-6736(20)30566-3

3. Pullano G, Di Domenico L, Sabbatini CE, et al. Underdetection of cases of COVID-19 in France threatens epidemic control. Nature. 2021;590(7844):134-139. doi:10.1038/s41586-020-03095-6

4. Wilkinson E. COVID-19 pandemic to lead to thousands of additional UK lung cancer deaths. Lancet Oncol. 2021;23:p23.

5. Rossi R, Socci V, Jannini TB, et al. Mental health outcomes among Italian health care workers during the COVID-19 pandemic. JAMA Network Open. 2021;4(11):e2136143. doi:10.1001/jamanetworkopen.2021.36143

6. Bryant-Genevier J, Rao CY, Lopes-Cardozo B, et al. Symptoms of Depression, Anxiety, Post-Traumatic Stress Disorder, and Suicidal Ideation Among State, Tribal, Local, and Territorial Public Health Workers During the COVID-19 Pandemic - United States, March-April 2021. MMWR Morb Mortal Wkly Rep. 2021;70 (48):1680-1685. doi:10.15585/mmwr.mm7048a6

7. Moya A, Serneels P, Desrosiers A, Reyes V, Torres MJ, Lieberman A. The COVID-19 pandemic and maternal mental health in a fragile and conflict-affected setting in Tumaco, Colombia: a cohort study. Lancet Global Health. 2021;9(8):e1068-e1076. doi:10.1016/S2214-109X (21)00217-5

8. Global prevalence and burden of depressive and anxiety disorders in. 204 countries and territories in 2020 due to the COVID-19 pandemic. Lancet. 2021;398(10312):1700-1712. doi:10.1016/S0140-6736(21) 02143-7

9. Razai MS, Oakeshott P, Kankam H, Galea S, Stokes-Lampard H. Mitigating the psychological effects of social isolation during the covid-19 pandemic. BMJ. 2020;369:m1904. doi:10.1136/bmj.m1904

10. Trudeau T. The Pygmalion effect in management. Hosp Top. 1980;58 (4):36. doi:10.1080/00185868.1980.9954800

11. Siekanska M, Blecharz J, Wojtowicz A. The athlete's perception of coaches' behavior towards competitors with a different sports level. J Hum Kinet. 2013;39:231-242. doi:10.2478/hukin-2013-0086

12. John MM. The "Pygmalion effect" and surgical mentoring. Indian J Surg. 2016;78(1):79. doi:10.1007/s12262-015-1432-3

13. Mai Z, Feng B, He Q, Feng Q. Medical thoracoscopic thermal ablation therapy for metastatic pleural tumors with malignant effusion: an Exploratory Retrospective Study. Int $J$ Gen Med. 2021;14:9349-9360. doi:10.2147/IJGM.S339596

14. Majumder J, Minko T. Recent developments on therapeutic and diagnostic approaches for COVID-19. AAPS J. 2021;23(1):14. doi:10.1208/s12248-020-00532-2

15. Kang H. Sample size determination and power analysis using the G Power software. J Educ Eval Health Prof. 2021;18:17. doi:10.3352/jeehp.2021.18.17

16. Köseoğlu TC, Demirbaș H, Bozkurt E, Acar H, Türk BÜ. Headache related to mask use of healthcare workers in COVID-19 pandemic. Korean J Pain. 2021;34(2):241-245. doi:10.3344/kjp.2021.34.2.241

17. Gennarelli SM, Brown SM, Mulcahey MK. Psychosocial interventions help facilitate recovery following musculoskeletal sports injuries: a systematic review. Phys Sportsmedicine. 2020;48(4):370-377. doi:10.1080/00913847.2020.1744486

18. Illi C, Brooke RI, Lau CL, Merskey H. Screening for psychiatric illness in patients with oral dysesthesia by means of the General Health Questionnaire-twenty-eight item version (GHQ-28) and the Irritability, Depression and Anxiety Scale (IDA). Oral Surg Oral Med Oral Pathol. 1989;67(4):384-389. doi:10.1016/0030-4220(89)90378-2
19. Qiu J, Guan J, Yang X, et al. Quality of Life and Psychological State in Chinese Breast Cancer Patients Who Received BRCA1/2 Genetic Testing. PLoS One. 2016;11(7):e0158531. doi:10.1371/journal. pone. 0158531

20. Qi W, Dong X, Xue X. The Pygmalion Effect to Piano Teaching From the Perspective of Educational Psychology. Front Psychol. 2021;12:690677. doi:10.3389/fpsyg.2021.690677

21. Nicoară-Farcău O, Wang X, Luo X. Definition of SPSS: we need to speak the same language. $J$ Hepatol. 2020;73(2):463-464. doi:10.1016/j.jhep.2020.03.012

22. Wang K, Goldenberg A, Dorison CA, et al. A multi-country test of brief reappraisal interventions on emotions during the COVID-19 pandemic. Nat Human Behav. 2021;5(8):1089-1110.

23. Liang L, Ren H, Cao R, et al. The Effect of COVID-19 on Youth Mental Health. Psychiatr Q. 2020;91(3):841-852. doi:10.1007/ s11126-020-09744-3

24. D'Emeh WM, Yacoub MI, Shahwan BS. Work-Related Stress and Anxiety Among Frontline Nurses During the COVID-19 Pandemic: a Cross-Sectional Study. J Psychosoc Nurs Ment Health Serv. 2021;59 (8):31-42. doi:10.3928/02793695-20210322-02

25. Sandín B, Espinosa V, Valiente RM, et al. Effects of Coronavirus Fears on Anxiety and Depressive Disorder Symptoms in Clinical and Subclinical Adolescents: the Role of Negative Affect, Intolerance of Uncertainty, and Emotion Regulation Strategies. Front Psychol. 2021;12:716528. doi:10.3389/fpsyg.2021.716528

26. Torres SJ, Nowson CA. Relationship between stress, eating behavior, and obesity. Nutrition. 2007;23(11-12):887-894. doi:10.1016/j. nut.2007.08.008

27. Mattioli AV, Nasi M, Cocchi C, Farinetti A. COVID-19 outbreak: impact of the quarantine-induced stress on cardiovascular disease risk burden. Future Cardiol. 2020;16(6):539-542. doi:10.2217/fca-20200055

28. Balez R, Leroyer C, Couturaud F. [Placebo effect: a contribution of social psychology]. Rev Mal Respir. 2014;31(8):714-720. doi:10.1016/j.rmr.2014.03.006. French.

29. Strength CB, Riche SC. The Pygmalion effect: nursing managers have the ability to stimulate performance. Nurs Success Today. 1986;3(9):15-17.

30. Bäuerle A, Teufel M, Musche V, et al. Increased generalized anxiety, depression and distress during the COVID-19 pandemic: a cross-sectional study in Germany. J Public Health. 2020;42 (4):672-678. doi:10.1093/pubmed/fdaa106

31. Burtscher J, Burtscher M, Millet GP. (Indoor) isolation, stress, and physical inactivity: vicious circles accelerated by COVID-19? Scand J Med Sci Sports. 2020;30(8):1544-1545. doi:10.1111/sms.13706

32. Bucciarelli V, Nasi M, Bianco F, et al. Depression pandemic and cardiovascular risk in the COVID-19 era and long COVID syndrome: gender makes a difference. Trends Cardiovasc Med. 2021. doi:10.1016/j.tcm.2021.09.009

33. Janjua S, Pike KC, Carr R, Coles A, Fortescue R, Batavia M. Interventions to improve adherence to pharmacological therapy for chronic obstructive pulmonary disease (COPD). Cochrane Database Sys Rev. 2021;9(9):Cd013381. doi:10.1002/14651858.CD013381. pub2

34. Borwell B. Continuity of care for the stoma patient: psychological considerations. Br J Community Nurs. 2009;14(8):326,328, 330-321. doi:10.12968/bjen.2009.14.8.43511

35. McPeake J, Pattison N. COVID-19: moving beyond the pandemic. $J$ Adv Nurs. 2020;76(10):2447-2449. doi:10.1111/jan.14438

36. Saha K, Torous J, Caine ED, De CM. Psychosocial Effects of the COVID-19 Pandemic: large-scale Quasi-Experimental Study on Social Media. J Med Internet Res. 2020;22(11):e22600. doi: $10.2196 / 22600$ 


\section{Publish your work in this journal}

The International Journal of General Medicine is an international, peer-reviewed open-access journal that focuses on general and internal medicine, pathogenesis, epidemiology, diagnosis, monitoring and treatment protocols. The journal is characterized by the rapid reporting of reviews, original research and clinical studies across all disease areas. The manuscript management system is completely online and includes a very quick and fair peer-review system, which is all easy to use. Visit http://www.dovepress.com/ testimonials.php to read real quotes from published authors. 